Association for Information Systems AIS Electronic Library (AISeL)

Wirtschaftsinformatik Proceedings 2003

Wirtschaftsinformatik

September 2003

\title{
Vorgehensmodell zur Evaluierung von Pervasive Computing Business Cases
}

Walter Niess

DaimlerChryslerAG, walter.niess@daimlerchrysler.com

Thomas Flor

DaimlerChrysler AG

Gabriel Vögler

DaimlerChrysler $A G$

Follow this and additional works at: http://aisel.aisnet.org/wi2003

\section{Recommended Citation}

Niess, Walter; Flor, Thomas; and Vögler, Gabriel, "Vorgehensmodell zur Evaluierung von Pervasive Computing Business Cases" (2003). Wirtschaftsinformatik Proceedings 2003. 9.

http://aisel.aisnet.org/wi2003/9

This material is brought to you by the Wirtschaftsinformatik at AIS Electronic Library (AISeL). It has been accepted for inclusion in Wirtschaftsinformatik Proceedings 2003 by an authorized administrator of AIS Electronic Library (AISeL). For more information, please contact elibrary@aisnet.org. 
In: Uhr, Wolfgang, Esswein, Werner \& Schoop, Eric (Hg.) 2003. Wirtschaftsinformatik 2003: Medien - Märkte - Mobilität, 2 Bde. Heidelberg: Physica-Verlag

ISBN: 3-7908-0111-9 (Band 1)

ISBN: 3-7908-0116-X (Band 2)

(C) Physica-Verlag Heidelberg 2003 


\title{
Vorgehensmodell zur Evaluierung von Pervasive Computing Business Cases
}

\author{
Walter Niess, Thomas Flor, Gabriel Vögler ${ }^{1}$ \\ DaimlerChrysler AG
}

\begin{abstract}
Zusammenfassung: Sollen Geschäftsprozesse (Business Cases) für mobile Anwendungen (Pervasive Computing) evaluiert werden, ist es notwendig, ein Portfolio von Anwendungsszenarien zusammenzustellen. Hierzu wird ein methodisches Vorgehen definiert, mit dessen Hilfe man einen priorisierten Überblick über mögliche Business Cases erhält sowie weitere Details hinsichtlich organisatorischer und technischer Aspekte untersucht. Aufgrund bestimmter Kriterien lassen sich Anwendungsszenarien auswählen, die für die Evaluierung herangezogen werden. Für diese wird ein ausführlicher Fragenkatalog erarbeitet, der als Entscheidungsgrundlage für die Realisierung der Business Cases dient.
\end{abstract}

Schlüsselworte: Pervasive Computing, Geschäftsprozesse, Requirements Engineering, Use Cases

\section{Mobile Anwendungen in Unternehmen}

Personal Digital Assistants (PDAs) finden immer größere Verbreitung. Wurden sie anfangs nur als elektronische Alternative eines papierbasierten Zeitplansystems zum Speichern persönlicher Daten, wie Kalender, Kontakte und Notizen verwendet, also sogenannter PIM(Personal Information Management)-Daten, so finden sich heute auch leistungsfähigere Anwendungen wie Textverarbeitung und Tabellenkalkulation.

Auch in Unternehmen finden PDAs immer breitere Anwendungsbereiche. Sie sind nicht nur als Einzelgerät oder offline einsetzbar, sondern werden zunehmend als Clients im verteilten Unternehmensnetzwerk integriert. Es ist möglich und im Einzelfall auch sinnvoll, größere Anwendungen auf ihnen auszuführen und auf Ressourcen im Unternehmensnetzwerk zurückzugreifen. So sind nicht nur die klassischen PIM-Daten auf mobilen Endgeräten verfügbar, sondern prinzipiell alle im Unternehmen bereitgestellten Informationen, wie z.B. Dateien auf Fileservern,

\footnotetext{
Herzlichen Dank an Herrn Thomas J. Uhl für seine große Hilfe bei der Erarbeitung des methodischen Vorgehens zur Evaluierung mobiler Anwendungen
} 
Inhalte von Datenbankservern und auch dynamisch von Applikations- oder Portalservern bereitgestellte Daten.

Um diese Informationen für mobile Endgeräte bereitstellen zu können, sind technische und organisatorische Maßnahmen notwendig, wie z.B. die Definition entsprechender Architekturen und das Bereitstellen von Diensten, wie z.B. Einwahlmöglichkeiten für mobile Geräte oder Synchronisationsdienste. Um all diese Maßnahmen durchzuführen, ist es Grundvoraussetzung, einen priorisierten Überblick über in Frage kommende mobile Anwendungen und Geschäftsprozesse zu erarbeiten. Die bereitgestellte Umgebung für Pervasive Computing muss vor Inbetriebnahme evaluiert werden. Hierzu ist es hilfreich, die Anwendungen nach einem definierten Schema zu kategorisieren, um das gesamte Anwendungsfeld und den technischen wie wirtschaftlichen Erfolg der einzelnen Business Cases im Einzelnen beurteilen zu können.

\section{Vorgehensmodell}

Alle Anwendungsfälle sollen nach einem bestimmten Schema mit den selben Fragestellungen beurteilt werden. Sie sind somit miteinander vergleichbar.

Für die Evaluierung der aufzubauenden Infrastruktur lassen sich Testfälle in Form eines Evaluierungsportfolios zusammenstellen. Es sollte möglichst viele unterschiedliche Testfälle enthalten, so dass alle Funktionen der zu testenden Infrastruktur evaluiert werden. Die Evaluierungsphase soll möglichst wenig Aufwand verursachen. Deshalb sollte das Evaluierungsportfolio Anwendungsfälle enthalten, die mit möglichst geringem technischen Aufwand umzusetzen sind, für die aber ein hoher wirtschaftlicher Erfolg (Business Impact) erwartet wird.

Durch ein schematisches Vorgehen soll es also möglich sein, Business Cases zu identifizieren, mit denen alle zu evaluierenden Funktionen des Pervasive Computing getestet werden können, die sowohl die Infrastruktur auf der mobilen Clientseite, auf der zunächst stationären Serverseite als auch im verbindenden Netzwerk einbeziehen.

\subsection{Vom Anwendungsfall zum Service Block}

Um die von einem Geschäftsprozess benötigten Funktionalitäten zu ermitteln, müssen zunächst die fachlichen Anforderungen (Business View) analysiert werden. Darauf basierend werden die technischen Anforderungen und deren Umsetzung (Technical View) identifiziert. Die benötigten technischen Funktionalitäten können abschließend zu Architekturkomponenten (Service Blocks) zusammengefasst werden, um für alle Geschäftsprozesse eine den Anforderungen entsprechend einheitliche Architektur für Pervasive Computing zu erstellen. 


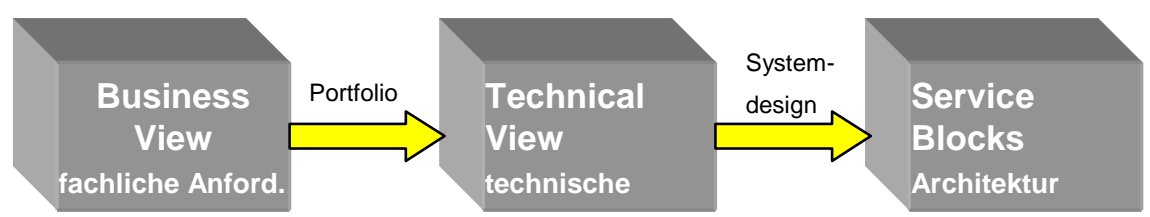

Abbildung 1: Business View, Technical View und daraus abgeleitete Service Blocks

Die identifizierten Architekturkomponenten stellen also alle in der Technical View definierten Funktionen zur Verfügung. Mit deren Hilfe lassen sich alle in der Business View ermittelten fachlichen Anforderungen realisieren.

Dieser Zusammenhang ist bei der Evaluierung von Bedeutung: Um Service Blocks zu evaluieren, werden Business Cases verwendet, die die querschnittlichen Funktionen der Service Blocks, wie z.B. Administration, Synchronisation, Transcoding, etc., verwenden. Die Business Cases eines Portfolios sind also so auszuwählen, dass die in den Technical Views der Business Cases beschriebenen Funktionen alle zu evaluierenden Funktionen der Service Blocks abdecken.

\section{Analyse von Pervasive Computing Business Cases}

Mobile Anwendungen können anhand von Fragekatalogen beurteilt werden. Je nach dem, welche Aspekte der Anwendungen beurteilt werden sollen, können diese mehr oder weniger umfangreich sein. So kann die Anwendung z.B. nur aus fachlicher Sicht oder auch deren technische Umsetzung betrachtet werden.

\subsection{Fachliche Anforderungen}

Die fachlichen Anforderungen eines Business Case können mit den folgenden Fragen ermittelt werden:

- Wer? Welcher Personenkreis verwendet die Anwendung? Sind weitere Personen durch den Betrieb der Anwendung betroffen, wie z.B. Administratoren oder Datenlieferanten?

- Was? Welche Daten soll der Anwender mit dem mobilen Business Case bearbeiten? Welche Informationen sollen von mobilen Endgeräten aus abgerufen werden können? Welche Daten werden modifiziert oder neu angelegt und müssen evtl. mit einem zentralen Datenserver abgeglichen werden? 
- Wann? Wann wird die mobile Anwendung verwendet? Zu welcher Tageszeit werden Informationen mit mobilen Endgeräten verarbeitet? Beschränkt sich dies auf bestimmte Zeiten oder ist es notwendig, den Datenzugriff rund um die Uhr zu ermöglichen?

- Wo? In welchem geographischen Bereich soll die mobile Anwendung eingesetzt werden? Muss sie nur auf dem Gelände des eigenen Unternehmens oder auch außerhalb der Unternehmens verfügbar sein? Ist eine Verfügbarkeit in Gebieten mit Mobilfunkabdeckung ausreichend?

Durch Klärung dieser Fragen wird der Anwendungsfall näher untersucht. Es wird die Business View der Anwendung ermittelt. Darauf aufbauend kann die Technical View ausgearbeitet werden.

\subsection{Template zur Klassifikation der Business Cases}

Um Business Cases für ein Portfolio auswählen zu können, ist es notwendig, neben den in der Business View ermittelten Informationen, auch Informationen über die mögliche technische Realisierung zu erhalten. Es empfiehlt sich, alle für den Auswahlprozess relevanten Informationen in Form eines Templates zu sammeln. Ein solches Template zur Klassifikation der einzelnen Business Cases beinhaltet also neben den Fragen, die sich mit der Business View beschäftigen, insbesondere Aspekte einer möglichen technischen Umsetzung.

Zur Evaluierung von Pervasive Computing Business Cases sind u.a. folgende Daten relevant. Sie können ggf. um weitere Aspekte ergänzt werden.

- Laufende Nummer des Business Case zur eindeutigen Identifikation

- Name des Business Case

- Kurzbeschreibung des Business Case: Allgemeine Beschreibung des Business Case und der Vorteile einer pervasiven Lösung

- Darstellung des möglichen synchronen Datenflusses im Business Case: Verbindungen, die immer aufgebaut sind und somit einen schnellen Zugang zu den Informationen gewährleisten. Meist mit höheren Kosten verbunden.

- Darstellung des möglichen asynchronen Datenflusses im Business Case: Verbindungen, die auf Wunsch aufgebaut und auch wieder abgebaut werden, wenn alle Informationen übertragen wurden.

- Darstellung des möglichen Push-Datenflusses im Business Case: Die Informationen werden automatisch an den Nutzer geschickt (z.B. per SMS).

- Darstellung des möglichen Pull-Datenflusses im Business Case: Der Nutzer prüft selbst, ob neue Informationen vorhanden sind.

- Zusätzliche Bemerkungen zum Business Case: z.B. ähnliche Einsatzfelder etc. 
- Differenzierungsmerkmal durch den Einsatz mobiler Endgeräte gegenüber stationären Lösungen: z.B. Zeitverzugslosigkeit, erhöhte Mobilität, einfachere Prozesse

- Wer benutzt den Business Case: z.B. Mitarbeiter, Kunden, Lieferanten

\begin{tabular}{|c|c|c|c|}
\hline \multicolumn{4}{|l|}{\begin{tabular}{|l|l|}
1. & E-Mail Zugang \\
\end{tabular}} \\
\hline $\begin{array}{l}\text { Beschreibung } \\
\text { Man kann unterwegs } \\
\text { an jedem beliebigen } \\
\text { Ort seine Mails lesen } \\
\text { und beantworten. } \\
\text { Durch die Aktualität } \\
\text { der Nachrichten ist } \\
\text { man ständig gut } \\
\text { informiert und kann } \\
\text { die elektronische Post } \\
\text { schneller und }\end{array}$ & $\begin{array}{l}\text { Synchroner Datenfluss } \\
\text { Man ist ständig online; das Mailprogramm } \\
\text { ruft in bestimmten kurzen Abschnitten } \\
\text { immer die E-Mails vom Server ab und man } \\
\text { bekommt die Nachricht sofort angezeigt } \\
\text { (analog SMS). Damit verbunden sind hohe } \\
\text { Telefonkosten. }\end{array}$ & \begin{tabular}{|l|} 
Asynchroner Datenfluss \\
Man kann seinen PDA veranlassen, die \\
Mails abzurufen, er baut eine \\
Wählverbindung auf, lädt die ausgewählten \\
Nachrichten herunter und trennt die \\
Verbindung wieder. Die Mail wird \\
beantwortet und zum Versenden kurz eine \\
Verbindung aufgebaut und nach dem \\
Senden eventuell nochmals auf neue Mails \\
geprüft und danach die Verbindung wieder \\
getrennt.
\end{tabular} & \multirow[t]{2}{*}{$\begin{array}{l}\text { Bemerkungen } \\
\text { Dies kann auch eine } \\
\text { fahrzeugspezifische } \\
\text { Anwendung sein. Das } \\
\text { Auto könnte } \\
\text { automatisch beim } \\
\text { Ensteigen nach } \\
\text { Identifikation der } \\
\text { serson den richtigen } \\
\text { Peail Account } \\
\text { abrufen. }\end{array}$} \\
\hline $\begin{array}{l}\text { effizienter bearbeiten } \\
\text { (z.B. in Pausen oder im } \\
\text { Stau). }\end{array}$ & $\begin{array}{l}\text { Push-Datenfluss } \\
\text { Es wird eine kurze Benachrichtigung } \\
\text { gesendet wenn eine Nachricht } \\
\text { angekommen ist und man kann sich die } \\
\text { Nachricht dann gezielt herunterladen. }\end{array}$ & $\begin{array}{l}\text { Pull-Datenfluss } \\
\text { Man schaut aktiv nach seinen Mails und } \\
\text { holt die Mails gezielt ab. }\end{array}$ & \\
\hline \multicolumn{4}{|c|}{$\begin{array}{l}\text { Differenzierungsmerkmal: } \\
\text { Zeitverzugslosigkeit, erhöhte Mobilität, Information reporting. }\end{array}$} \\
\hline \multicolumn{2}{|c|}{$\begin{array}{l}\text { Wer (People): } \\
\text { Alle Mitarbeiter, die eine Bürotätigkeit ausüben. }\end{array}$} & \multirow{2}{*}{\multicolumn{2}{|c|}{$\begin{array}{l}\text { Device Support } \\
\text { SmartPhone, PDA, mobile Notebook. Um die E-Mails lesen und } \\
\text { auch beantworten zu können, wird ein graphisches Display } \\
\text { benötigt. }\end{array}$}} \\
\hline \multicolumn{2}{|l|}{$\begin{array}{l}\text { Wo (Location): } \\
\text { Weltweit. }\end{array}$} & & \\
\hline \multicolumn{2}{|c|}{$\begin{array}{l}\text { Wann (Time + Anforderungen Verfügbarkeit): } \\
7 \text { Tage / } 24 \text { Stunden Verfügbarkeit }\end{array}$} & \multirow{2}{*}{\multicolumn{2}{|c|}{$\begin{array}{l}\text { Erforderliche Bandbreite } \\
\text { für reine Text E-Mails reicht GSM. }\end{array}$}} \\
\hline \multicolumn{2}{|c|}{$\begin{array}{l}\text { Was (Information + durchschnittliche Datenmenge pro } \\
\text { Transaktion): } \\
\text { Zugang zum Mailserver und damit zur Nachricht. Datenmenge etwa } \\
1 \text { - } 10 \text { KB pro E-Mail, zuzüglich mögliche Attachments. }\end{array}$} & & \\
\hline \multicolumn{2}{|c|}{$\begin{array}{l}\text { Anforderungen Performanz: Gering } \\
\text { Die E-Mail sollte sofort ankommen (insbesondere ein Hinweis auf } \\
\text { eine neu eingegangene E-Mail), kann aber beim Herunterladen } \\
\text { Verzögerungen verkraften. }\end{array}$} & \multicolumn{2}{|c|}{$\begin{array}{l}\text { Anforderungen Umsetzbarkeit: } \\
\text { relativ wenig Aufwand, da die Technik nur noch auf Wreless } \\
\text { adaptiert werden muss. }\end{array}$} \\
\hline \multicolumn{2}{|c|}{$\begin{array}{l}\text { Anforderungen Datensicherheit: Normal } \\
\text { Es werden unter Umständen wichtige Daten transferiert, welche } \\
\text { man durch Verwendung von Verschlüsselung (z.B. PGP) schützen } \\
\text { kann. }\end{array}$} & \multicolumn{2}{|c|}{$\begin{array}{l}\text { Business Impact (durch zusätzlichen Ensatz mobiler } \\
\text { Endgeräte): Mittel } \\
\text { Höhere Kundenzufriedenheit durch Eleichterung des } \\
\text { Arbeitsprozesses. }\end{array}$} \\
\hline
\end{tabular}

Abbildung 2: Beispiel-Template für den Business Case E-Mail-Zugang

- Wo wird der Business Case genutzt: mögliche Nutzungsbereiche, bevorzugter Einsatzort, Einschränkungen

- Wann wird der Business Case genutzt: mögliche und bevorzugte Einsatzzeit und -häufigkeit

- Was wird im Business Case benutzt: Information und Datenmenge

- Device Support: Welche mobilen Endgeräte können zum Einsatz kommen

- Erforderliche Bandbreite zur Übertragung der Informationen im Business Case: hieraus kann abgeleitet werden, welche Mobilfunktechnologie sich für die 
Realisierung eignet ( $\leq$ 14,4 Kbps: GSM, $\leq 56 \mathrm{Kbps}$ : HSCSD, $\leq 171,2 \mathrm{Kbps}$ : GPRS, $\leq 192$ Kbps: EDGE, $\leq 2$ Mbps: UMTS, $\leq 56$ Mbps: WLAN). Selbstverständlich sind hierbei auch weitere Eigenschaften der Mobilfunktechnologien zu beachten.

- Anforderungen des Business Case an die Performanz: Reaktionszeiten des Systems, erwartete Belastungen der Netzwerk- und Serverinfrastruktur

- Anforderungen des Business Case an die Datensicherheit: Schutz sicherheitsrelevanter Daten, falls solche übertragen werden

- Anforderungen des Business Case an die Umsetzbarkeit: Einschätzung des technischen und organisatorischen Aufwands zur Business Case-Realisierung

- Business Impact des Business Case: Einschätzung der erhofften Vorteile und des wirtschaftlichen Erfolgs bei Business Case-Realisierung

Detailliertere Beschreibungen der Implementierung sind in dieser Stufe der Business Case Analyse nicht notwendig. Die Templates können bei Bedarf zur Definition einer geeigneten Umsetzungsarchitektur iterativ verfeinert werden.

\subsection{Portfolio der analysierten Business Cases}

Das Portfolio zur Evaluierung und Priorisierung der wichtigsten Business Cases wird nach vorher festzulegenden Kriterien erstellt. Da hierbei in der Regel mehrere Beurteilungsaspekte relevant sind, ist der Auswahlprozess nicht trivial. Um die Auswahl von Business Cases zu vereinfachen, können z.B. grafische Darstellungen verwendet werden.

Sollen zunächst in einer Evaluationsphase beispielsweise mit möglichst wenig Aufwand die für das Unternehmen relevanten Business Cases realisiert werden, so kann ein Portfolio sinnvoll nach folgenden Kriterien zusammengestellt werden:

- Mit den Business Cases sollen alle zu evaluierenden Funktionen getestet werden können, d.h. die Business Cases sollen möglichst einen hohen Anteil querschnittlicher Funktionen (Service Blocks) verwenden.

- Die Business Cases sollen mit möglichst geringem Aufwand umzusetzen sein.

- Die Business Cases sollen einen möglichst hohen Business Impact haben. Hierunter sind prinzipiell alle Auswirkungen auf Geschäftsabläufe des Unternehmens zu verstehen, insbesondere die Einsparung von Zeit und finanziellen Mitteln sowie Erhöhung der Produktqualität. Dies kann z.B. durch Vereinfachung oder Verbesserung von IT-gestützten Geschäftsprozessen bei Verwendung mobiler Technologien erreicht werden. 
Da die Größen Aufwand und Business Impact i.d.R. erst nach erfolgter Implementierung sichtbar sind und auch dann u.U. nur schwer messbar sind, können sie im Vorfeld lediglich abgeschätzt werden.

Die Kriterien können je nach durchzuführender Evaluation variieren und sind im Einzelfall festzulegen.

Mit einem zweidimensionalen Portfolio (Darstellung wesentlicher Evaluierungsparameter eines Geschäftsprozesses in Beziehung zueinander) kann die Auswahl der Business Cases vereinfacht werden. Um z.B. Business Cases mit hohem Business Impact und geringem Umsetzungsaufwand zu ermitteln, sollten diese beiden Parameter dargestellt werden.

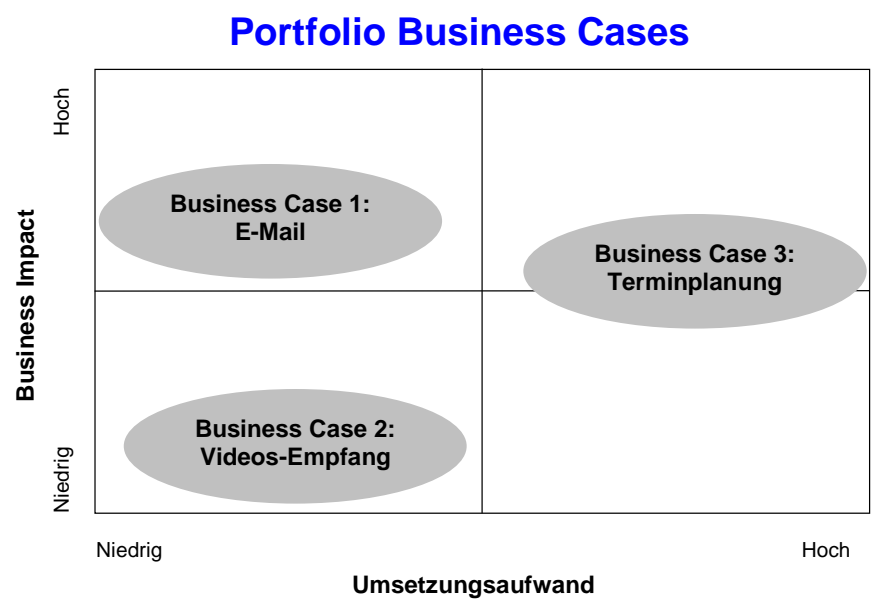

Abbildung 3: Portfolio mit Umsetzungsaufwand und Business Impact

Da in der Regel nicht nur zwei Parameter für die Auswahl relevant sind, kann eine solche zweidimensionale Darstellung lediglich eine Hilfe für den Auswahlprozess darstellen. Alternativ sind mehrdimensionale Portfolios zu erstellen bzw. mehrere Portfolios in Beziehung zueinander zu setzen.

\section{Analyse der fachlichen und technischen Anforderungen}

Für die Umsetzung der mittels Portfolio priorisierten Business Cases werden zusätzliche Informationen benötigt. Um diese zu ermitteln, wird ein ausführlicher und einheitlicher Fragenkatalog zu jedem umzusetzenden Business Case erarbeitet. Er bildet auf Basis der Templates eine stark verfeinerte Entscheidungsgrund- 
lage für die Auswahl, der für die Realisierung zu verwendenden Architekturkomponenten und Technologien.

Bei diesem Fragebogen werden neben detaillierteren Business-Aspekten die funktionalen Anforderungen, die technischen Randbedingungen sowie organisatorische Aspekte betrachtet:

- Business-Aspekte:

Durch die Analyse weiterer Business-Aspekte ist der ökonomische Hintergrund besser zu verstehen. Dadurch soll sichergestellt werden, dass beim Design der technischen Lösung stets die wirtschaftlichen Aspekte im Vordergrund stehen.

- Zunächst wird die Mission beschrieben, die mit Realisierung des Business Case verfolgt wird. Dazu gehören Gründe, weshalb die angestrebte mobile Lösung benötigt wird und was mit ihr bezweckt werden soll.

- Weiterhin werden die gewünschten Ziele festgehalten. Für die Kontrolle der Zielerreichung müssen messbare Kriterien definiert werden, wie z.B. bestimmte finanzielle Veränderungen oder messbare Verbesserungen der Zufriedenheit von Anwendern oder Kunden gegenüber einer evtl. bereits bestehenden stationären Lösung.

- Ausserdem sollten alle beteiligten Personen ermittelt werden, also neben Anwendern auch Administratoren, Datenlieferanten, etc. Da u.U. durch eine mobile Lösung nicht nur Vorteile zu erwarten sind, sollte ermittelt werden, ob die Personen von der Lösung profitieren oder ob sich Nachteile ergeben. Solche Nachteile können z.B. ein höherer Administrations- oder Datenpflegeaufwand sein.

- Wichtig ist klarzustellen, welche Veränderung in definierten Prozessabläufen zu erwarten sind, wenn mobile Technologien zur Umsetzung von Geschäftsprozessen verwendet werden.

- Funktionale Anforderungen:

Vor dem Hintergrund der ermittelten Business-Aspekte werden die funktionalen Anforderungen verfeinert. Sie dienen als Basis für den Entwurf der technischen Lösung und bestimmen die für die Umsetzung relevanten Architekturkomponenten. Ferner ergibt sich ein erster Überblick über die im System verarbeiteten Informationen und die notwendigen Datenflüsse.

- Es werden die mit der Anwendung zu erledigenden Aufgaben ermittelt. Zu jeder Aufgabe wird festgehalten, welche Benutzergruppe diese zu erledigen hat. Neben den Endbenutzern werden auch Systembenutzer etc. betrachtet.

- Für jeden Arbeitsablauf werden die einzelnen Arbeitsschritte dargestellt. 
- Daraus ergibt sich auch eine Übersicht der benötigten Daten. Es wird dargestellt, wer bzw. welche technische Komponente welche Daten verarbeitet und woher diese kommen. Daraus ergeben sich Schnittstellen zu externen Systemen, mit denen Daten ausgetauscht werden.

- Weiterhin kann die Wichtigkeit bestimmter Systemeigenschaften, wie z.B. Performanz, Sicherheit oder Diensteverfügbarkeit abgeschätzt werden.

- Technische Randbedingungen:

Alle einzuhaltende Randbedingungen und Architekturvorgaben sowie die bereits vorhandene technische Infrastruktur (Server, mobile Clients, bestehendes Netzwerk) werden dokumentiert. Dazu gehören:

- die vorhandene Infrastruktur, wie Systemumgebungen, Betriebssystemplattformen, Middleware-Systeme und Testumgebungen

- Unternehmensstandards, wie z.B. Systemmanagement und Betrieb von Anwendungen

- Netzwerkzugänge und Einwahlknoten

- Sicherheitsstandards.

- Organisatorische Aspekte:

Mit der Einführung mobiler Geschäftsprozesse sind gewisse organisatorische Aspekte verbunden, wie z.B.

- Planung

- Betrieb

- Verlauf eines Rollouts.

Der erarbeitete Fragenkatalog, aus dem mit der obigen Auflistung nur ein Überblick der wichtigsten Aspekte dargestellt wurde, analysiert den Business View und den Technical View sehr detailliert. Mit diesen Informationen läßt sich einerseits die Beurteilung und Priorisierung der Business Cases im Portfolio iterativ überarbeiten als auch andereseits die geplante Realisierung der Business Cases zielgerichteter umsetzen.

\section{Zusammenfassung}

Ist für die Evaluierung und Priorisierung von Pervasive Computing Business Cases ein Portfolio zu erarbeiten, so müssen zunächst die für die Auswahl relevanten Beurteilungskriterien systematisch ermittelt werden. Hierzu gehört neben wesentlichen Aspekten, wie geringer Umsetzungsaufwand oder hoher Business Impact, 
die Abdeckung der zu evaluierenden Funktionen durch den einzelnen Business Case. Ein systematisches Vorgehen wird durch Ableiten der Technical View aus der Business View und anschließendem Abgleich mit den Funktionen der notwendigen Service Blocks sichergestellt. Alle für die Zusammenstellung des Portfolios relevanten Beurteilungskriterien werden mit einem Template erfasst.

Zur Durchführung der Evaluierung sind weitere Details der Geschäftsprozesse nötig, die in einem verfeinerten Fragenkatalog beantwortet werden.

Durch dieses iterative Top-Down-Vorgehensmodell werden die für den jeweiligen Schritt einer Umsetzung von Pervasive Computing Business Cases notwendigen Informationen zielgerichtet erarbeitet und auch unter wirtschaftlichen Gesichtspunkten ausgewertet.

\section{Literatur}

[HaHe01] Hausmann, J.H.; Heckel: Use Cases as views: A formal approach to Requirements engineering in the Unified Process. Informatik 2001: Tagungsbang der GI/OCGJahrestagung, 25.-28. September 2001, Universität Wien, Band 1, S. 595-599

[JoMa96] Johnson, D.B.; Maltz, D.A.: Protocols for Adaptive Wireless and Mobile Networking. IEEE Personal Communications Magazine 3 No. 1, 1996, S. 34-41

[KuGu00] Kulak, D.; Guiney, E.: Use Cases: Requirements in Context. Addison Wesley Professional: Boston, MA, 2000

[Somm01] Sommerville, I.: Software Engineering, 6. Auflage. Addison Wesley Professional: Boston, MA, 2001

[Zöll02] Zöller-Greer, P.: Softwareengineering für Ingenieure und Informatiker. Vieweg Verlag: Wiesbaden, 2002 